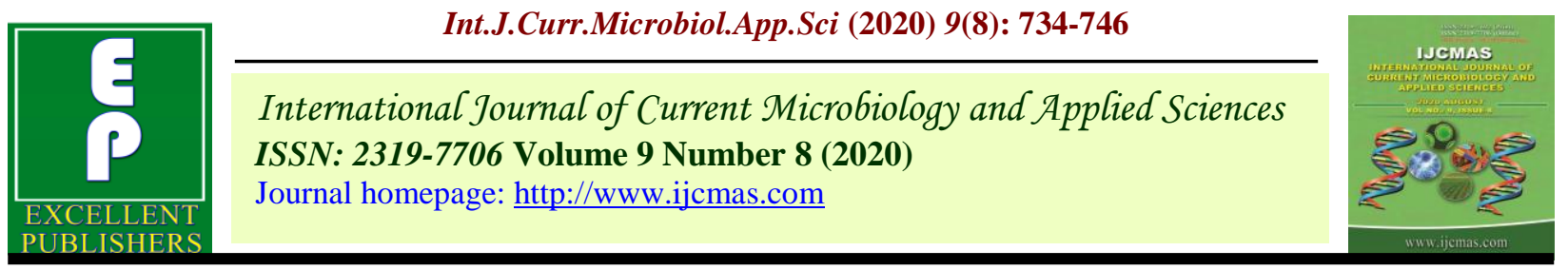

Original Research Article

https://doi.org/10.20546/ijcmas.2020.908.079

\title{
Revival of Channapatna Toy Motifs on Textiles through Digital Printing
}

\author{
R. Rashmi and Sadhana D. Kulloli* \\ Department of Textile and Apparel Designing, College of Community Science, University of \\ Agricultural Sciences, Dharwad-580005, Karnataka, India \\ *Corresponding author
}

\section{A B S T R A C T}

\section{Keywords}

Channapatna toys, Motifs, consumer acceptance, Border designs

\section{Article Info}

Accepted:

10 July 2020 Available Online:

10 August 2020
The present study was carried out to collect the popular and available motifs of Channapatna toys, develop Channapatna toy motifs on textiles through digital printing and to know the consumer acceptance of textiles printed with Channapatna toy motifs. Based on primary and secondary data, the available fifty different Channapatna toys were collected. Out of fifty motifs, textile experts selected thirty motifs suitable for textile printing and out of thirty motifs, fifteen each single motif and combination of motif border designs of sizes 1", 2 " and 3" were designed totaling to 45 border designs. Totally twelve border designs, two each of 1", 2" and 3" sizes of both single and combination of motif border designs were selected based on the highest Weighted Mean Score (WMS) rated by the textile experts based on motif clarity, colour combination and overall appearance suitable for digital printing on three types of fabrics viz., cotton poplin, coarse cotton and crepe silk satin. The acceptability index was found to be highest in case of combination of motif border design on coarse cotton of 2" size $\left(\mathrm{DC}_{\mathrm{C} 2.2}, 92.22 \%\right)$ followed by 3 " size $\left(\mathrm{DC}_{\mathrm{C} 3.2,}, 91.11 \%\right)$ and single motif border design of 3" size $\left(\mathrm{DS}_{\mathrm{C} 3.2,}, 90 \%\right)$.

\section{Introduction}

India is endowed with the rich heritage in designing and printing of clothes. Today, textile designs are used by everyone for making beautiful garments. Textile designing has been an ancient art and perhaps the most ancient craft of India. This is the most demanding and emerging field as it has full of scope and creativity. The design sources may include nature, famous monuments, and various kinds of toys, folk arts and crafts (Sharma et al., 2014). Motifs can be used for value addition of textile products with proper adaptation for surface enrichment techniques such as embroidery, batik printing, screen printing, digital printing, block printing and weaving. In order to fulfill the requirement of ever changing market, the designers constantly look for new colour, style, motifs and designs (Anjum et al., 2014).

Printing is one of the most preferred techniques to create interest in woven fabrics. Extraordinary remarkable designs can be developed by using different printing techniques viz., screen and digital printing. Digital printing is also known as direct to 
garment printing. Digital printing is a process of printing on textiles and garments using specialized or modified inkjet technology. It produces images, colours and patterns by using computer controlled inkjet nozzles.

It's environmentally excellent because the inks are water based and very little water is required for washing off and also as all ink goes on to the fibre and none into the waste water. Therefore, to save the environment from pollution, use of such techniques should be encouraged; hence textile designing with digital printing is an eco-friendly solution (Goel et al., 2012).

Channapatna toy, a play material can also be a motif on the textile material as one of the value addition. Consumers are the one who always look for new designs for fashion purpose. As Channapatna toy motif creates emphasis, energy urge to use a new alteration (varieties of garment) it is planned to create ready to use borders for the ultimate use on children garments in order to bring change in design, a new approach through which this Channapatna toy as a motif can be explored and also one of the way to sustain the traditional Indian art.

Preparation of ready to use borders through digital printing by the implication of the Channapatna toys as the main motifs, would add variety to the existing designs of borders. Thus, the study is planned to explore various motifs from Channapatna toys, to adapt these motifs for digital printing by CATD technique and applying on textiles by way of producing ready to use borders and to know the consumer acceptance of ready to use borders printed with Channapatna toy motifs.

\section{Materials and Methods}

The information on the artisans of Channapatna town of Ramanagar district was personally interviewed to collect the information on the type of Channapatna toys produced and the colour combinations used. Fifty Channapatna toy motifs were collected. Out of 50 motifs, textile experts selected 30 motifs based on their suitability for textile printing.

From these thirty motifs, fifteen single motif and fifteen combinations of motif borders were designed, totaling to thirty different borders using Corel draw software. These thirty borders were further designed into three different sizes viz, 1", 2" and 3". Finally thirty Textile experts were asked to rate these different sized borders (single and combination) based on their suitability, motif clarity, colour and size.

Out of three different sized borders from fifteen each single and combination of borders, the highest two rankings from each size of single ( 6 borders) and combination of motifs (6 borders) were selected (totally 12 borders) for development of digital printed ready to use borders. Three different types of fabrics, viz cotton poplin (fine), coarse cotton and crepe silk satin material were selected for digital printing separately to know the printing effect on different materials.

To know the acceptance of the digital printed Channapatna toy motifs on textile materials used as ready to use borders, thirty respondents (textile experts and housewives) were selected from Dharwad. The samples of digital printed textiles with Channapatna toy motifs in three different fabrics were displayed to know its relevance, suitability, motif clarity and colour combination.

Further, to assess the percentage acceptability of digital printed ready to use borders, an Acceptability Index (A.I) was calculated (Upadhayay and Babel, 2013). 


\section{Results and Discussion}

A total of 50 collected Channapatna toy motifs were collected from the artisans of Channapatna toy manufacturers and internet (https://in.pinterest.com/saurabhroys/channap atna-toys). A questionnaire containing five point rating scale (Excellent, Very Good, Good, Fair and Poor) was distributed to thirty textile experts to rate the fifty collected Channapatna toy motifs (Table1). A total of thirty motifs were selected by the textile experts based on suitability of motifs for textile application.

The highest Weighted Mean Score was given to wooden top (4.79) followed by to wooden ducks (4.59), wooden piggy set (4.58), abacus rattle (4.55), woman pounding (4.53), woman churning milk and wooden horse rider (4.52) and were ranked from 1 to 6 accordingly. However, the least was given to wooden rabbit car (2.06) and fish toy (2.1).

Out of these thirty selected Channapatna toy motifs, fifteen single motif border designs and fifteen combinations of Channapatna toy motif border designs of three sizes (1", 2" and 3 ") were created and developed in computer using Corel Draw software.

They were coded as $S_{1}$ to $S_{15}$ for single border designs and $\mathrm{C}_{1}$ to $\mathrm{C}_{15}$ for combination of motif border designs (Table 2 and Plate 1). Thus, a total of 45 single motif border designs and 45 combinations of motif border designs totaling to 90 border designs were created.

Textile experts rated these ninety border designs based on its motif clarity and combinations, colour combination and overall appearance using five point rating scale. It is seen from Table 2 that textile experts rated highest (Weighted Mean Score) to $S_{1}$ (4.93) followed by $\mathrm{S}_{12}$ (4.16) for 1" single motif border design, $S_{2}$ (4.35) followed by $S_{7}$ (4.33) for 2" and for 3" $\mathrm{S}_{11}$ (4.49) followed by $\mathrm{S}_{3}$ (4.40) designs.

While the highest rating for 1"combination of motif border designs were given to $\mathrm{C}_{13}$ (4.36) followed by to $\mathrm{C}_{9}$ (4.26), for 2", $\mathrm{C}_{11}$ (4.38) followed by $\mathrm{C}_{8}$ (4.12) and for 3 " $\mathrm{C}_{15}$ (4.83) followed $\mathrm{C}_{4}$ (4.16) were rated highest. Thus, the above 12 border designs (6 each from single motif and combination of motif border designs) taking two each form all three sizes viz., 1", 2" and 3" were selected for digital printing on textile materials and coded as $\mathrm{S}_{1.1}$, $\mathrm{S}_{1.2}, \mathrm{~S}_{1.3}, \mathrm{~S}_{2.1}, \mathrm{~S}_{2.2}, \mathrm{~S}_{2.3}, \mathrm{~S}_{3.1}, \mathrm{~S}_{3.2}, \mathrm{~S}_{3.3}$ (Plate 2a) and $\mathrm{C}_{1.1}, \mathrm{C}_{1.2}, \mathrm{C}_{1.3}, \mathrm{C}_{2.1}, \mathrm{C}_{2.2}, \mathrm{C}_{2.3}, \mathrm{C}$ ${ }_{3.1}, C_{3.2}, C_{3.3}$ (Plate 2b).

Cotton poplin, coarse cotton and crepe silk satin cloth are the three types of fabrics selected for digital printing because these are the materials popularly used for printing. For comparison these fabrics were selected to know the difference in the clarity of the motifs in digital printing.

The selected twelve Channapatna toy motif designs suitable for ready to use borders were digitally printed on cotton poplin, coarse cotton and crepe silk satin on three different types of selected fabrics using reactive and acidic inks for cotton and crepe silk satin fabrics respectively.

Based on the type of fabric and size of motif, the digital printed ready to use borders were coded as $\mathrm{DS}_{\mathrm{P} 1.1}, \mathrm{DS}_{\mathrm{P} 1.2}, \mathrm{DS}_{\mathrm{P} 2.1}, \mathrm{DS}_{\mathrm{P} 2.2,} \mathrm{DS}_{\mathrm{P} 3.1}$, $\mathrm{DS}_{\mathrm{P} 3.2}, \mathrm{DCp}_{1.1}, \mathrm{DC}_{\mathrm{P} 1.2 .} \mathrm{DCp}_{2.1}, \mathrm{DC}_{\mathrm{P} 2.2,}, \mathrm{DCp}_{3.1}$, $\mathrm{DC}_{\mathrm{P} 3.2,} \mathrm{DS}_{\mathrm{C} 1.1}, \mathrm{DS}_{\mathrm{C} 1.2}, \mathrm{DS}_{\mathrm{C} 2.1}, \mathrm{DS}_{\mathrm{C} 2.2,} \mathrm{DS}_{\mathrm{C} 3.1}$, $\mathrm{DS}_{\mathrm{C} 3.2}, \mathrm{DCc}_{1.1}, \mathrm{DC}_{\mathrm{c} 1.2 .} \mathrm{DCc}_{2.1}, \mathrm{DC}_{\mathrm{c} 2.2,}, \mathrm{DCc}_{3.1}$, $\mathrm{DC}_{\mathrm{c} 3.2}$ and $\mathrm{DS}_{\mathrm{s} 1.1,}, \mathrm{DS}_{\mathrm{s} 1.2}, \mathrm{DS}_{\mathrm{s} 2.1}, \mathrm{DS}_{\mathrm{s} 2.2,} \mathrm{DS}_{\mathrm{s} 3.1}$, $\mathrm{DS}_{\mathrm{s} 3.2}, \mathrm{DCs}_{1.1}, \mathrm{DC}_{\mathrm{s} 1.2 .} \mathrm{DCs}_{2.1}, \mathrm{DC}_{\mathrm{s} 2.2}, \mathrm{DCs}_{3.1}$, $\mathrm{DC}_{\mathrm{s} 3.2}$ (Table 3a, 3b and 3c) 
Table.1 WMS for selection of Channapatna toy motifs suitable on textiles

\begin{tabular}{|c|c|c|c|}
\hline Sl. No. & Motif's & WMS & RANK \\
\hline 1 & Wooden $1-5$ ring set stacker & 3.65 & 19 \\
\hline 2 & Wooden piggy set & 4.58 & 03 \\
\hline 3 & Wooden joker ringset & 4.08 & 13 \\
\hline 4 & Wooden taboo ringset & 4.46 & 07 \\
\hline 5 & Wooden bear ringset & 3.62 & 20 \\
\hline 6 & Wooden cup and ball & 3.75 & 18 \\
\hline 7 & Bell rattles & 3.36 & 25 \\
\hline 8 & Wooden ring o rattle & 3.10 & 26 \\
\hline 9 & Wooden single turtle & 4.22 & 09 \\
\hline 10 & Wooden joker car & 3.79 & 17 \\
\hline 11 & Woman churning milk & 4.52 & 06 \\
\hline 12 & Woman's pounding & 4.53 & 05 \\
\hline 13 & Wooden umbrella women & 3.99 & 14 \\
\hline 14 & Farmer & 3.59 & 21 \\
\hline 15 & Abacus rattle & 4.55 & 04 \\
\hline 16 & Dumbbells rattle & 3.96 & 15 \\
\hline 17 & Wooden top & 4.79 & 01 \\
\hline 18 & Wooden egg shaker & 2.19 & 38 \\
\hline 19 & Wooden magic top & 2.85 & 29 \\
\hline 20 & Wooden bird toy & 2.79 & 30 \\
\hline 21 & Wooden rings toy & 3.36 & 25 \\
\hline 22 & Wooden joker & 4.03 & 11 \\
\hline 23 & Wooden car & 3.92 & 16 \\
\hline 24 & Wooden elephant & 3.10 & 26 \\
\hline 25 & Tommy wooden toy & 2.33 & 38 \\
\hline 26 & Wooden helicopter & 2.33 & 38 \\
\hline 27 & Wooden train engine & 3.37 & 24 \\
\hline 28 & Wooden tractor & 2.47 & 35 \\
\hline 29 & Wooden scooter & 3.01 & 28 \\
\hline 30 & Wooden joker helicopter & 3.53 & 22 \\
\hline 31 & Wooden movable simile toy & 2.65 & 32 \\
\hline 32 & Wooden butterfly & 4.15 & 10 \\
\hline 33 & Wooden fish toy & 2.10 & 42 \\
\hline 34 & Wooden rabbit car & 2.06 & 41 \\
\hline 35 & Wooden plate tic toc & 2.60 & 40 \\
\hline 36 & Wooden plate rattle & 2.75 & 31 \\
\hline 37 & Wooden egg rattle-maracas & 2.29 & 37 \\
\hline 38 & Wooden flower tik tak & 2.49 & 34 \\
\hline 39 & Wooden waggy dog & 4.02 & 12 \\
\hline 40 & Wooden peddling duck & 2.38 & 36 \\
\hline 41 & Wooden frog ratchet & 2.53 & 33 \\
\hline 42 & Wooden war weapon & 2.79 & 30 \\
\hline 43 & Wooden ducks & 4.59 & 02 \\
\hline 44 & Wooden duck top & 3.42 & 23 \\
\hline 45 & Wooden whistles & 2.48 & 34 \\
\hline 46 & Wooden key chain & 3.09 & 27 \\
\hline 47 & Wooden Ganesha & 4.00 & 13 \\
\hline 48 & Wooden bullock cart & 4.46 & 07 \\
\hline 49 & Wooden elephant & 4.29 & 08 \\
\hline 50 & Wooden horse rider & 4.52 & 06 \\
\hline
\end{tabular}


Table.2 Weighted mean score for preference of single and combination of Channapatna toy motif border designs

\begin{tabular}{|c|c|c|c|c|}
\hline \multirow[t]{2}{*}{ Sl. No } & \multirow[t]{2}{*}{ Borders } & \multicolumn{3}{|c|}{ WMS } \\
\hline & & $1 "$ & $2 "$ & 3" \\
\hline 1 & S1 & 4.93 & 4.00 & 3.68 \\
\hline 2 & $\mathbf{S 2}$ & 3.96 & 4.35 & 4.20 \\
\hline 3 & S3 & 2.96 & 4.00 & 4.40 \\
\hline 4 & S4 & 4.03 & 4.14 & 4.12 \\
\hline 5 & S5 & 3.09 & 3.86 & 3.45 \\
\hline 6 & S6 & 2.83 & 4.13 & 3.82 \\
\hline 7 & S7 & 3.69 & 4.33 & 4.15 \\
\hline 8 & S8 & 3.69 & 3.96 & 3.9 \\
\hline 9 & S9 & 3.83 & 4.00 & 4.16 \\
\hline 10 & S10 & 3.93 & 4.16 & 2.96 \\
\hline 11 & S11 & 4.09 & 4.15 & 4.49 \\
\hline 12 & S12 & 4.16 & 3.51 & 3.06 \\
\hline 13 & S13 & 3.09 & 4.15 & 4.10 \\
\hline 14 & S14 & 3.65 & 3.75 & 3.89 \\
\hline 15 & S15 & 3.83 & 4.22 & 4.12 \\
\hline 16 & C1 & 3.08 & 3.35 & 2.99 \\
\hline 17 & $\mathrm{C} 2$ & 3.65 & 3.49 & 3.29 \\
\hline 18 & C3 & 4.06 & 3.29 & 3.46 \\
\hline 19 & C4 & 3.89 & 3.65 & 4.16 \\
\hline 20 & C5 & 3.46 & 3.46 & 3.29 \\
\hline 21 & C6 & 3.42 & 3.45 & 3.49 \\
\hline 22 & C7 & 3.45 & 3.39 & 3.03 \\
\hline 23 & C8 & 3.49 & 4.12 & 3.39 \\
\hline 24 & C9 & 4.26 & 4.08 & 3.89 \\
\hline 25 & C10 & 3.82 & 3.85 & 3.59 \\
\hline 26 & C11 & 3.55 & 4.38 & 3.66 \\
\hline 27 & C12 & 3.76 & 3.86 & 3.72 \\
\hline 28 & C13 & 4.36 & 3.89 & 3.99 \\
\hline 29 & C14 & 3.52 & 3.21 & 3.52 \\
\hline 30 & C15 & 4.15 & 4.06 & 4.83 \\
\hline
\end{tabular}

S1-S15: Single motif border designs of Channapatna toys

C1-C15: Combination motif border designs of Channapatna toys 
Table.3 (a) Weighted mean score for the acceptance of digital printed ready to use border designs with Channapatna toy motifs on cotton poplin fabric

\begin{tabular}{|c|c|c|c|c|}
\hline Coding & Code details & $\begin{array}{l}\text { Motif } \\
\text { clarity }\end{array}$ & $\begin{array}{c}\text { Colour } \\
\text { combination }\end{array}$ & $\begin{array}{c}\text { Overall } \\
\text { appearance }\end{array}$ \\
\hline DS $_{\mathbf{P 1 . 1}}$ & Digital printed single motif border design on cotton poplin of 1" & 2.69 & 2.98 & 3.69 \\
\hline $\mathbf{D S}_{\mathbf{P 1 . 2}}$ & Digital printed single motif border design on cotton poplin of 1 " & 3.12 & 4.19 & 2.29 \\
\hline $\mathbf{D S}_{\mathbf{P 2 . 1}}$ & Digital printed single motif border design on cotton poplin of 2" & 4.06 & 3.92 & 4.22 \\
\hline $\mathbf{D S}_{\mathbf{P 2 . 2}}$ & Digital printed single motif border design on cotton poplin of 2" & 3.98 & 3.96 & 3.98 \\
\hline $\mathbf{D S}_{\mathbf{P 3 . 1}}$ & Digital printed single motif border design on cotton poplin of 3" & 4.59 & 4.14 & 4.36 \\
\hline $\mathrm{DS}_{\mathbf{P 3 . 2}}$ & Digital printed single motif border design on cotton poplin of $3 "$ & 4.22 & 3.98 & 3.96 \\
\hline DC $_{\text {P1.1 }}$ & Digital printed combination of motif border design on cotton poplin of 1 " & 3.98 & 4.22 & 4.19 \\
\hline DC $_{\text {P1.2 }}$ & Digital printed combination of motif border design on cotton poplin of 1" & 4.52 & 4.36 & 4.15 \\
\hline $\mathbf{D C}_{\mathbf{P 2 . 1}}$ & Digital printed combination of motif border design on cotton poplin of 2" & 4.12 & 4.19 & 4.19 \\
\hline $\mathbf{D C}_{\mathbf{P 2 . 2}}$ & Digital printed combination of motif border design on cotton poplin of 2" & 4.56 & 4.22 & 4.08 \\
\hline $\mathbf{D C}_{\mathbf{P 3 . 1}}$ & Digital printed combination of motif border design on cotton poplin of 3" & 4.76 & 3.99 & 3.69 \\
\hline $\mathbf{D C}_{\mathbf{P 3 . 2}}$ & Digital printed combination of motif border design on cotton poplin of 3" & 4.59 & 4.53 & 4.19 \\
\hline
\end{tabular}

Table.3 (b) Weighted mean score for the acceptance of digital printed ready to use border designs with Channapatna toy motif on coarse cotton fabric

\begin{tabular}{|c|c|c|c|c|}
\hline Coding & Code details & $\begin{array}{l}\text { Motif } \\
\text { clarity }\end{array}$ & $\begin{array}{l}\text { Colour } \\
\text { combination }\end{array}$ & $\begin{array}{c}\text { Overall } \\
\text { appearance }\end{array}$ \\
\hline $\mathbf{D S}_{\mathbf{C 1 . 1}}$ & Digital printed single motif border design on coarse cotton of 1" & 3.78 & 3.75 & 3.85 \\
\hline $\mathbf{D S}_{\mathbf{C} 1.2}$ & Digital printed single motif border design on coarse cotton of 1" & 3.78 & 4.02 & 3.72 \\
\hline $\mathbf{D S}_{\mathbf{C 2 . 1}}$ & Digital printed single motif border design on coarse cotton of $2 "$ & 4.03 & 2.66 & 4.8 \\
\hline $\mathbf{D S}_{\mathrm{C} 2.2}$ & Digital printed single motif border design on coarse cotton of $2 "$ & 4.69 & 4.09 & 4.82 \\
\hline $\mathbf{D S}_{\mathbf{C} 3.1}$ & Digital printed single motif border design on coarse cotton of 3" & 4.06 & 4.06 & 4.29 \\
\hline $\mathbf{D S}_{\mathbf{C} 3.2}$ & Digital printed single motif border design on coarse cotton of 3" & 4.96 & 4.52 & 4.46 \\
\hline $\mathrm{DC}_{\mathbf{C 1 . 1}}$ & $\begin{array}{l}\text { Digital printed combination of motif border design on coarse } \\
\text { cotton of 1" }\end{array}$ & 3.56 & 3.02 & 4.52 \\
\hline $\mathrm{DC}_{\mathbf{C 1 . 2}}$ & $\begin{array}{l}\text { Digital printed combination of motif border design on coarse } \\
\text { cotton of 1" }\end{array}$ & 4.15 & 4.35 & 4.13 \\
\hline $\mathrm{DC}_{\mathrm{C} 2.1}$ & $\begin{array}{l}\text { Digital printed combination of motif border design on coarse } \\
\text { cotton of 2" }\end{array}$ & 4.56 & 2.82 & 4.39 \\
\hline $\mathrm{DC}_{\mathrm{C} 2.2}$ & $\begin{array}{l}\text { Digital printed combination of motif border design on coarse } \\
\text { cotton of 2" }\end{array}$ & 4.46 & 4.56 & 4.66 \\
\hline $\mathrm{DC}_{\mathrm{C} 3.1}$ & $\begin{array}{l}\text { Digital printed combination of motif border design on coarse } \\
\text { cotton of 3" }\end{array}$ & 3.5 & 4.29 & 4.05 \\
\hline $\mathrm{DC}_{\mathrm{C} 3.2}$ & $\begin{array}{l}\text { Digital printed combination of motif border design on coarse } \\
\text { cotton of 3" }\end{array}$ & 4.46 & 4.63 & 4.56 \\
\hline
\end{tabular}


Table.3 (c) Weighted mean score for the acceptance of digital printed ready to use border designs with Channapatna toy motifs on crepe silk satin fabric

\begin{tabular}{|c|c|c|c|c|}
\hline Coding & Code details & Motif clarity & $\begin{array}{c}\text { Colour } \\
\text { combination }\end{array}$ & $\begin{array}{c}\text { Overall } \\
\text { appearance }\end{array}$ \\
\hline $\mathrm{DS}_{\mathrm{S} 1.1}$ & $\begin{array}{l}\text { Digital printed single motif border design on crepe silk } \\
\text { satin of 1" }\end{array}$ & 3.8 & 4.16 & 4.19 \\
\hline $\mathbf{D S}_{\mathrm{S} 1.2}$ & $\begin{array}{l}\text { Digital printed single motif border design on crepe silk } \\
\text { satin of 1" }\end{array}$ & 3.92 & 3.22 & 4.25 \\
\hline $\mathrm{DS}_{\mathrm{S} 2.1}$ & $\begin{array}{l}\text { Digital printed single motif border design on crepe silk } \\
\text { satin of 2" }\end{array}$ & 4.36 & 3.72 & 4.56 \\
\hline $\mathrm{DS}_{\mathrm{S} 2.2}$ & $\begin{array}{l}\text { Digital printed single motif border design on crepe silk } \\
\text { satin of 2" }\end{array}$ & 3.53 & 3.92 & 3.99 \\
\hline $\mathbf{D S}_{\mathrm{S} 3.1}$ & $\begin{array}{l}\text { Digital printed single motif border design on crepe silk } \\
\text { satin of 3" }\end{array}$ & 4.56 & 4.22 & 3.96 \\
\hline $\mathrm{DS}_{\mathrm{S} 3.2}$ & $\begin{array}{l}\text { Digital printed single motif border design on crepe silk } \\
\text { satin of 3" }\end{array}$ & 4.46 & 3.89 & 3.92 \\
\hline $\mathrm{DC}_{\mathrm{S} 1.1}$ & $\begin{array}{l}\text { Digital printed combination of motif border design on } \\
\text { crepe silk satin of 1" }\end{array}$ & 4.00 & 3.86 & 4.36 \\
\hline $\mathrm{DC}_{\mathrm{S} 1.2}$ & $\begin{array}{l}\text { Digital printed combination of motif border design on } \\
\text { crepe silk satin of } 1 \text { " }\end{array}$ & 4.49 & 3.76 & 4.22 \\
\hline $\mathrm{DC}_{\mathrm{S} 2.1}$ & $\begin{array}{l}\text { Digital printed combination of motif border design on } \\
\text { crepe silk satin of } 2 \text { " }\end{array}$ & 3.92 & 4.55 & 2.98 \\
\hline $\mathrm{DC}_{\mathrm{S} 2.2}$ & $\begin{array}{l}\text { Digital printed combination motif border design on } \\
\text { crepe silk satin of } 2 "\end{array}$ & 3.99 & 4.52 & 4.22 \\
\hline $\mathrm{DC}_{\mathrm{S3.1}}$ & $\begin{array}{l}\text { Digital printed combination of motif border design on } \\
\text { crepe silk satin of } 3 \text { " }\end{array}$ & 3.72 & 4.15 & 4.52 \\
\hline $\mathrm{DC}_{\mathrm{S} 3.2}$ & $\begin{array}{l}\text { Digital printed combination of motif border design on } \\
\text { crepe silk satin of } 3 \text { " }\end{array}$ & 4.59 & 4.25 & 4.57 \\
\hline
\end{tabular}

Table.4 (a) Acceptability Index of the digital printed ready to use border designs with Channapatna toy motifs on cotton poplin fabric

\begin{tabular}{|c|c|c|c|c|c|c|c|c|c|c|c|c|c|}
\hline \multirow[t]{2}{*}{ SL. No } & \multirow[t]{2}{*}{ Parameters } & \multicolumn{12}{|c|}{ Mean score } \\
\hline & & DS $_{\mathbf{P 1 . 1}}$ & $\mathbf{D S}_{\mathrm{P} 1.2}$ & $\mathbf{D S}_{\mathbf{P} 2.1}$ & $\mathbf{D S}_{\mathbf{P} 2.2}$ & DS $_{\mathbf{P} 3.1}$ & DS $_{\mathbf{P} 3.2}$ & $\mathrm{DC}_{\mathbf{P 1 . 1}}$ & $\mathrm{DC}_{\mathbf{P 1 . 2}}$ & $\mathrm{DC}_{\mathbf{P 2 . 1}}$ & $\mathrm{DC}_{\mathbf{P} 2.2}$ & $\mathrm{DC}_{\mathbf{P 3 . 1}}$ & $\mathrm{DC}_{\mathbf{P} 3.2}$ \\
\hline 1 & $\begin{array}{l}\text { Motif } \\
\text { clarity }\end{array}$ & 19 & 19 & 24 & 24 & 28 & 25 & 24 & 27 & 25 & 28 & 28 & 28 \\
\hline 2 & $\begin{array}{l}\text { Colour } \\
\text { combination }\end{array}$ & 18 & 25 & 24 & 24 & 26 & 24 & 25 & 26 & 25 & 25 & 24 & 27 \\
\hline 3 & $\begin{array}{l}\text { Overall } \\
\text { appearance }\end{array}$ & 28 & 14 & 25 & 26 & 24 & 24 & 25 & 26 & 25 & 25 & 22 & 25 \\
\hline \multicolumn{2}{|c|}{$\begin{array}{l}\text { Total scores obtained } \\
\text { by the border designs }\end{array}$} & 65 & 58 & 73 & 74 & 78 & 73 & 74 & 79 & 75 & 78 & 74 & 80 \\
\hline \multicolumn{2}{|c|}{ Acceptability Index } & 72.22 & 64.44 & 81.11 & 82.22 & 86.67 & 81.11 & 82.22 & 87.78 & 83.33 & 86.67 & 82.22 & 88.88 \\
\hline
\end{tabular}


Table.4 (b) Acceptability Index of the digital printed ready to use border designs with Channapatna toy motifs on coarse cotton fabric

\begin{tabular}{|c|c|c|c|c|c|c|c|c|c|c|c|c|c|}
\hline \multirow{2}{*}{$\begin{array}{l}\text { SL. } \\
\text { No }\end{array}$} & \multirow[t]{2}{*}{ Parameters } & \multicolumn{12}{|c|}{ Mean score } \\
\hline & & $\mathbf{D S}_{\mathbf{C} 1.1}$ & $\mathbf{D S}_{\mathrm{C} 1.2}$ & $\mathbf{D S}_{\mathbf{C} 2.1}$ & $\mathrm{DS}_{\mathrm{C} 2.2}$ & $\mathbf{D S}_{\mathbf{C 3 . 1}}$ & $\mathrm{DS}_{\mathrm{C} 3.2}$ & $\mathrm{DC}_{\mathrm{C} 1.1}$ & $\mathrm{DC}_{\mathrm{C} 1.2}$ & $\mathrm{DC}_{\mathrm{C} 2.1}$ & $\mathrm{DC}_{\mathrm{C} 2.2}$ & $\mathrm{DC}_{\mathrm{C3.1}}$ & $\mathrm{DC}_{\mathrm{C} 3.2}$ \\
\hline 1 & $\begin{array}{l}\text { Motif } \\
\text { clarity }\end{array}$ & 23 & 23 & 24 & 28 & 28 & 27 & 21 & 25 & 28 & 27 & 25 & 27 \\
\hline 2 & $\begin{array}{l}\text { Colour } \\
\text { combination }\end{array}$ & 23 & 24 & 21 & 25 & 24 & 27 & 18 & 26 & 21 & 28 & 26 & 28 \\
\hline 3 & $\begin{array}{l}\text { Overall } \\
\text { appearance }\end{array}$ & 23 & 22 & 24 & 21 & 26 & 27 & 27 & 21 & 26 & 28 & 24 & 27 \\
\hline \multicolumn{2}{|c|}{$\begin{array}{l}\text { Total scores } \\
\text { obtained by the } \\
\text { border designs }\end{array}$} & 69 & 69 & 69 & 74 & 78 & 81 & 66 & 76 & 75 & 83 & 75 & 82 \\
\hline & $\begin{array}{l}\text { ceptability } \\
\text { Index }\end{array}$ & 76.67 & 76.67 & 76.67 & 82.22 & 86.67 & 90.00 & 73.33 & 84.44 & 83.33 & 92.22 & 83.33 & 91.11 \\
\hline
\end{tabular}

Table.4(c) Acceptability Index of the digital printed ready to use border designs with Channapatna toy motifs on crepe silk satin fabric

\begin{tabular}{|c|c|c|c|c|c|c|c|c|c|c|c|c|c|}
\hline \multirow{2}{*}{$\begin{array}{l}\text { SL } \\
\text { No }\end{array}$} & \multirow[t]{2}{*}{ Parameters } & \multicolumn{12}{|c|}{ Mean score } \\
\hline & & $\mathrm{DS}_{\mathrm{S} 1.1}$ & $\mathrm{DS}_{\mathbf{S} 1.2}$ & $\mathrm{DS}_{\mathrm{S} 2.1}$ & $\mathrm{DS}_{\mathrm{S} 2.2}$ & $\mathrm{DS}_{\mathbf{S} 3.1}$ & $\mathrm{DS}_{\mathrm{S} 3.2}$ & $\mathrm{DC}_{\mathrm{S} 1.1}$ & $\mathrm{DC}_{\mathrm{S} 1.2}$ & $\mathrm{DC}_{\mathrm{S} 2.1}$ & $\mathrm{DC}_{\mathrm{S} 2.2}$ & $\mathrm{DC}_{\mathrm{S} 3.1}$ & $\mathrm{DC}_{\mathrm{S} 3.2}$ \\
\hline 1 & $\begin{array}{l}\text { Motif } \\
\text { clarity }\end{array}$ & 23 & 24 & 26 & 21 & 27 & 27 & 24 & 21 & 24 & 24 & 22 & 28 \\
\hline 2 & $\begin{array}{l}\text { Colour } \\
\text { combination }\end{array}$ & 25 & 19 & 22 & 24 & 21 & 23 & 23 & 22 & 27 & 27 & 25 & 26 \\
\hline 3 & $\begin{array}{l}\text { Overall } \\
\text { appearance }\end{array}$ & 25 & 26 & 28 & 24 & 24 & 24 & 26 & 25 & 18 & 25 & 27 & 27 \\
\hline & $\begin{array}{l}\text { otal scores } \\
\text { ained by the } \\
\text { der designs }\end{array}$ & 73 & 69 & 78 & 69 & 72 & 74 & 73 & 68 & 69 & 76 & 74 & 81 \\
\hline & $\begin{array}{l}\text { ceptability } \\
\text { Index }\end{array}$ & 81.11 & 76.67 & 86.67 & 76.67 & 80.00 & 82.22 & 81.11 & 75.56 & 76.67 & 84.44 & 82.22 & 90.00 \\
\hline
\end{tabular}


Plate.1 Selected Channapatna toy motifs for creation of borders

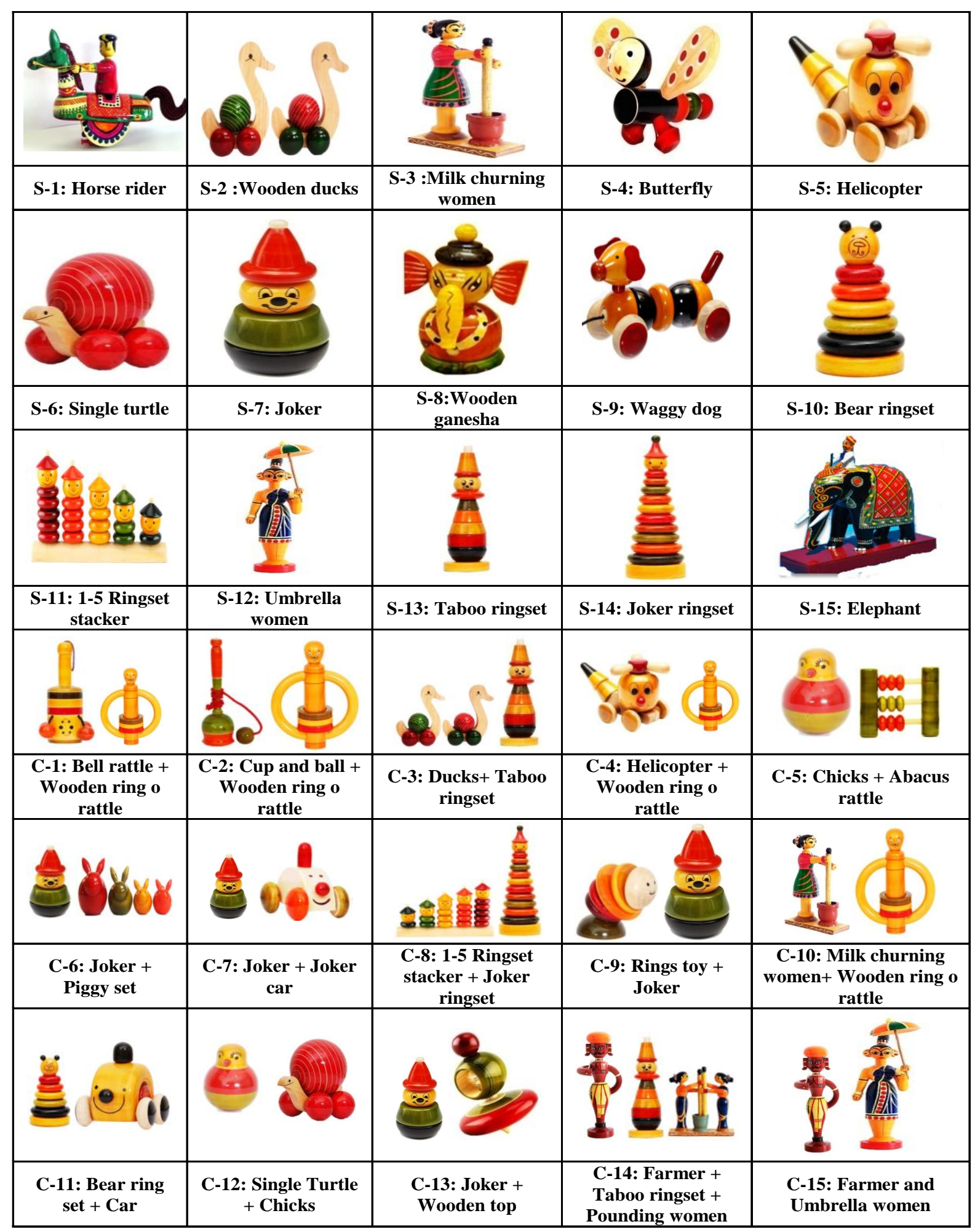


Plate.2a Single Channapatna toy motif border designs

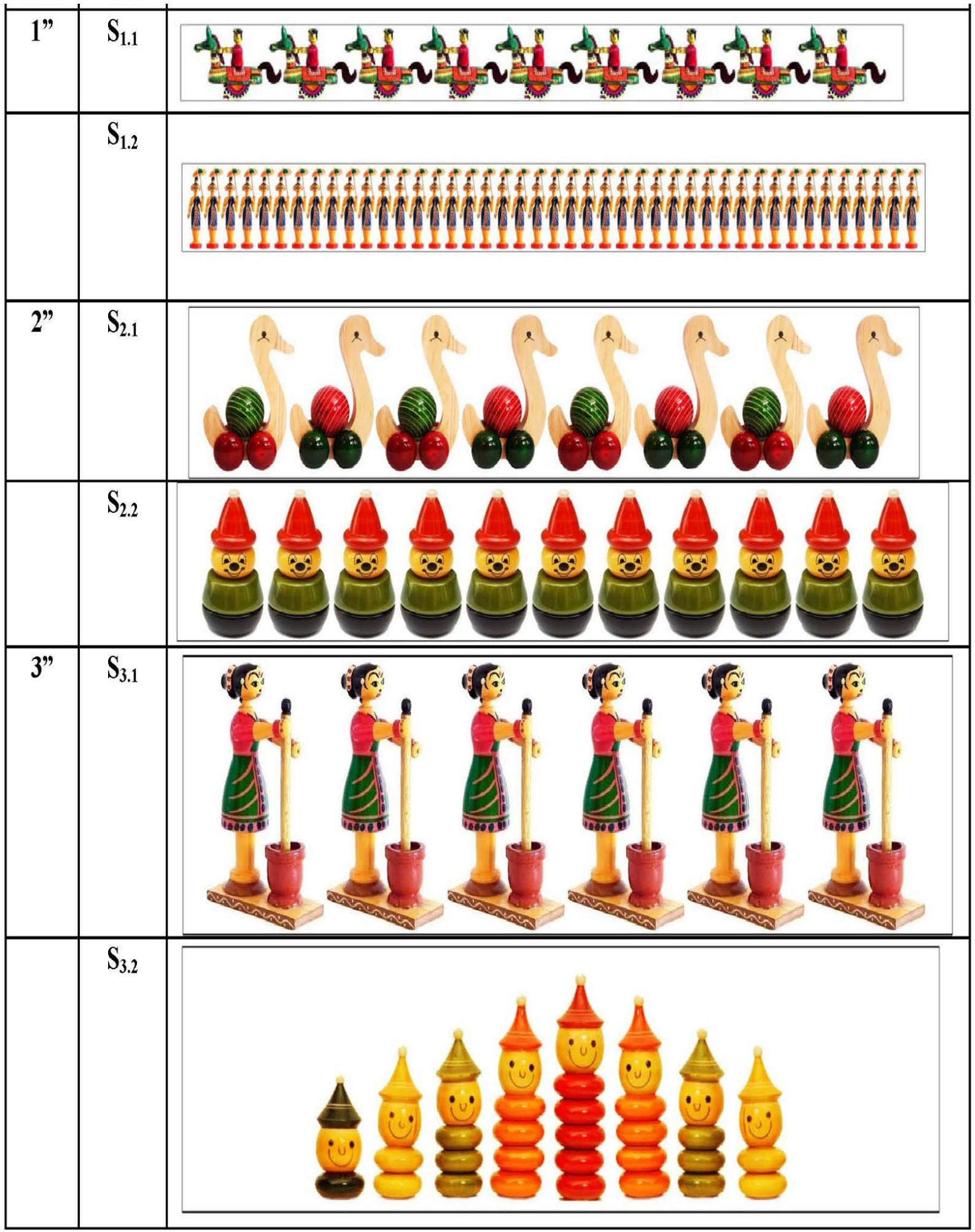


Plate.2b Combination of Channapatna toy motif border designs

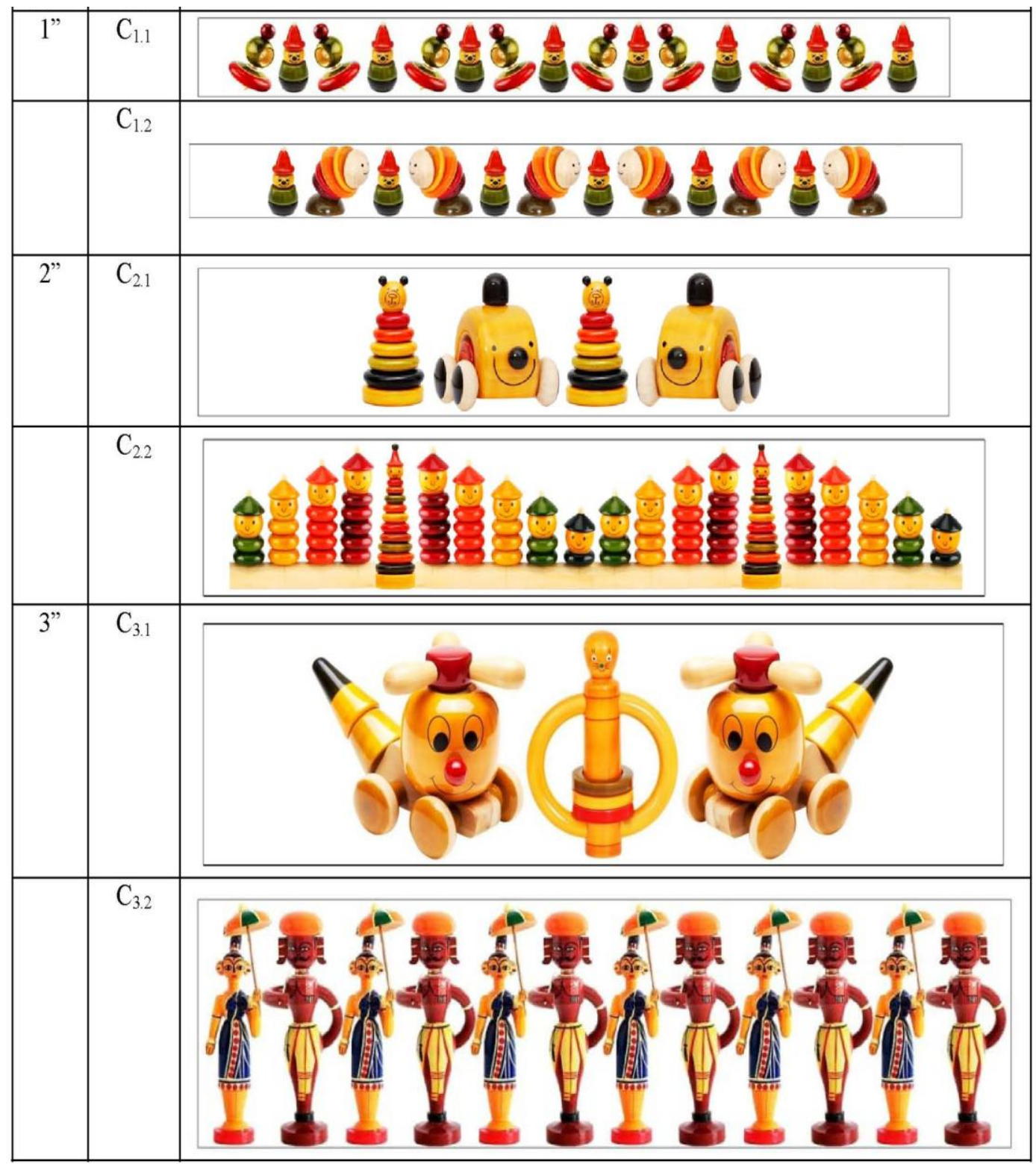

Acceptance of ready to use digital printed borders with Channapatna toy motifs

Majority of the respondents rated highest for $\mathrm{DS}_{\mathrm{P} 3.1}$ (4.59) for motif clarity in single border design, $\mathrm{DS}_{\mathrm{P} 1.2}$ (4.19) for colour combination and $\mathrm{DS}_{\mathrm{P} 3.1}$ (4.36) for overall appearance of digital printed ready to use border with Channapatna toy motifs on Cotton poplin fabric (Table 3a). However, for combination of motifs designs, $\mathrm{DC}_{\mathrm{P} 3.1}$ (4.76) was rated highest for motif clarity, $\mathrm{DC}_{\mathrm{P} 3.2}$ (4.53) for colour combination and $\mathrm{DC}_{\mathrm{P} 1.1}, \mathrm{DC}_{\mathrm{P} 2.1}$ and $\mathrm{DC}_{\mathrm{P} 3.2}$ (4.19) for overall appearance.

The acceptance of ready to use borders printed on coarse cotton with Digital printing by the respondents is presented in Table $3 \mathrm{~b}$. It is found from the table that the highest WMS for single motif border designs were rated for $\mathrm{DS}_{\mathrm{C} 3.2}$ (4.96 and 4.52) in case of motif clarity and colour combination respectively and for 
overall appearance highest WMS is for $\mathrm{DS}_{\mathrm{C} 2.2}$ (4.82). However, in case of combination of motifs, the highest rating was given for $\mathrm{DC}_{\mathrm{C} 2.1}$ (4.56) border for motif clarity, $\mathrm{DC}_{\mathrm{C} 3.2}$ (4.63) border for colour combination and $\mathrm{DC}_{\mathrm{C} 2.2}$ (4.66) for overall appearance of digital printing. Table $3 \mathrm{c}$ reveals about the acceptance of ready to use borders on crepe silk satin fabric. Respondents rated highest for $\mathrm{DS}_{\mathrm{S} 3.1}$ (4.56) \& $\mathrm{DC}_{3.2}$ (4.59) for motif clarity, $\mathrm{DS}_{\mathrm{S} 3.1}$ (4.22) \& $\mathrm{DC}_{\mathrm{S} 2.1}$ (4.55) for colour combination and $\mathrm{DS}_{\mathrm{S} 2.1}$ (4.56) \& $\mathrm{DC}_{\mathrm{S} 3.2}$ (4.57) for overall appearance for both single and combination of motif border designs respectively printed in different sizes.

Irrespective of three types of fabrics, respondents rated highest for $\mathrm{DS}_{\mathrm{C} 3.2}$ (WMS4.96 and 4.52) border for motif clarity and colour combination in case of single motif border designs and overall appearance for $\mathrm{DS}_{\mathrm{C} 2.2}$ (4.82) border. However, in case of combination of motif border designs, the highest WMS was rated for $\mathrm{DC}_{\mathrm{P} 3.1}$ (4.76) for motif clarity, $\mathrm{DC}_{\mathrm{C} 3.2}$ (4.63) for colour combination and $\mathrm{DC}_{\mathrm{C} 2.2}$ (4.66) for overall appearance of the motifs on the borders. Irrespective of fabrics and single or combination of motifs, motif clarity was better in $\mathrm{DS}_{\mathrm{C} 3.2}$ (4.96) border, colour combination was found to be better in $\mathrm{DC}_{\mathrm{C} 3.2}$ (4.63) border and overall appearance was rated highest for $\mathrm{DS}_{\mathrm{C} 2.2}$ (4.82) border design.

The acceptability index of digital printed ready to use borders with Channapatna toy motifs in case of single motif border design was found to be higher in $\operatorname{DS}_{\mathrm{P} 3.1}(86.67 \%)$ and $\mathrm{DC}_{\mathrm{P} 3.2}(88.88 \%)$ in case of combination of motif border designs (Table $4 a, 4 b$ and $4 c$ ). The acceptability index on coarse cotton in case of single motif digital printed border designs was found to be highest in $\mathrm{DS}_{\mathrm{C} 3.2}(90$ $\%)$ and $\mathrm{DC}_{\mathrm{C} 2.2}(92.22 \%)$ in case of combination of motif border designs. In case of crepe silk satin fabric, the highest acceptability index was for $\operatorname{DS}_{\mathrm{S} 2.1}(86.67 \%)$ for single motif border design and $\mathrm{DC}_{\mathrm{S} 3.2}(90$ $\%$ ) for combination of border designs.

Irrespective of type of fabrics used, the highest acceptability index was found in $\mathrm{S}_{\mathrm{C} 3.2}$ (90\%) and $\mathrm{DC}_{\mathrm{C} 2.2}(92.22 \%)$ in single motif and combination of motif border designs respectively because of motif clarity, colour combination and overall appearance.

In conclusion the highest acceptability index was found better on coarse cotton digitally printed with combination of 1-5 ringset stacker and joker ringset Channapatna toy motifs, hence considered most suitable Channapatna toy motifs for textile printing. Thus, printing with Channapatna toy motif is a new approach and creative material for children. The motifs can also printed with different colour combinations, motif combinations and on different materials to bring variety in printed materials for children garments and bedlinens. Preparation of ready to use borders with the Channapatna toy motifs through digital printing is a new approach through which an traditional art can be revived and explored for textile designing.

\section{References}

Anjum, S., Archana, S. and Shweta, T., 2014, Development of innovative abstract motifs for screen printing. Asian $J$. Home Sci., 9 (1):264-266.

Goel, B., Singh R and Pant G.B., 2012. Digital inkjet printing solution to textile designing, Man-made Tex. India., 40 (7): 265-268.

Sharma, E., Amanpreet, K. and Susan, P., 2014, Digitalization of Madhubani designs for transferring on apparels using screen printing technique. Eduved Int. J. Interdisciplinary Res., 1 (7):1-12.

Upadhayay, H. and Banel,S.,2013,Magic of 
floor on fabric: Revival of traditional floor printing of Kumaon by contemporary adaptation of Apparels. Asian J.Home Sci.,8(2):722-725. https://in.pinterest.com/saurabhroys/channapa tna-toys

\section{How to cite this article:}

Rashmi, R. and Sadhana D. Kulloli. 2020. Revival of Channapatna Toy Motifs on Textiles through Digital Printing. Int.J.Curr.Microbiol.App.Sci. 9(08): 734-746.

doi: https://doi.org/10.20546/ijcmas.2020.908.079 